\title{
Scalarization and robustness in uncertain vector optimization problems: a non componentwise approach
}

\author{
Elisa Caprari ${ }^{1} \cdot$ Lorenzo Cerboni Baiardi $^{2}$ (D) Elena Molho ${ }^{1}$
}

Received: 29 December 2020 / Accepted: 31 January 2022 / Published online: 21 February 2022

(c) The Author(s) 2022

\begin{abstract}
The robust optimization approach can be used to tackle uncertain vector problems by considering worst case scenarios. In this context, notions of robust efficient solutions which are coherent with a set-valued minimization process have been introduced in literature in order to avoid unduly pessimistic attitudes (see e.g. Ehrgott et al. in Eur. J. Oper. Res. 239(1), 17-31, 2014). We address the question whether scalarization and robustification can be commuted in a non componentwise framework. We prove that the commutation of the two approaches is ensured under appropriate assumptions. To this purpose, we identify a class of scalarization processes that ensure necessary and sufficient robust optimality conditions through the direct scalarization of the uncertain vector optimization problem, without explicitly passing through the set-valued formulation of the problem.
\end{abstract}

Keywords Vector optimization · Robust optimization · Scalarization · Uncertainty

\section{Introduction}

Vector optimization problems, where objectives depend on uncertain parameters, can be tackled by using the robust approach. Such a methodology aims at hedging the decision maker against worst case scenarios that may occur as the uncertainties vary within their domains. Moreover, it is suitable to face the situation where no probability distribution on parameters is available. The robust approach, introduced by [28] (see also [3]) and widely developed in [2] for scalar cases, can be extended to uncertain vector optimization programs.

Lorenzo Cerboni Baiardi

lorenzo.cerboni@unibo.it

Elisa Caprari

elisa.caprari@unipv.it

Elena Molho

elena.molho@unipv.it

1 Department of Economics and Management, University of Pavia, Via San Felice 5, +39 27100 Pavia, Italy

2 Department of Mathematics, University of Bologna, Piazza Porta San Donato 5, +3940126 Bologna, Italy 


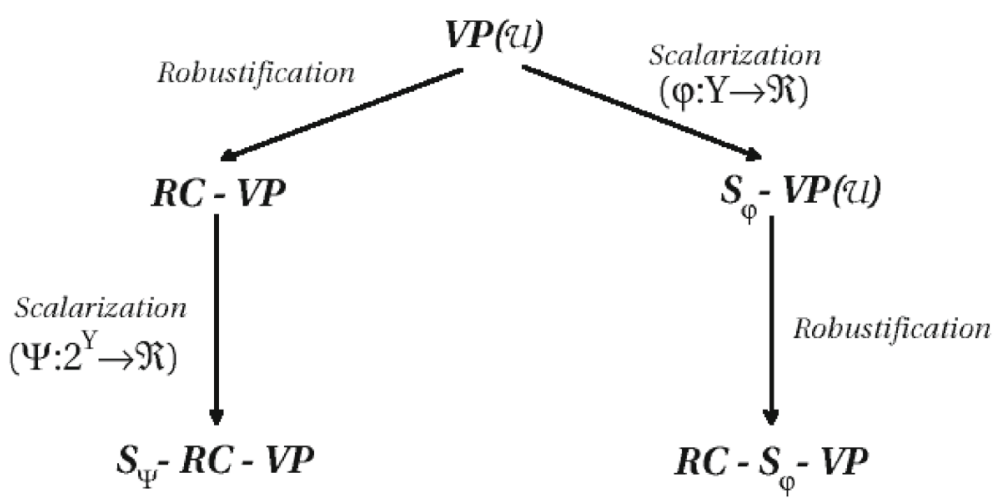

Fig. 1 Scalarization and robustification

The simplest way to introduce robustness in the parametric multiobjective optimization problem is to consider the worst case scenario on each component of the multicriteria objective function. It is the so called componentwise approach introduced in [27] and considered, for example, in $[8,10]$. This approach is unduly restrictive when interdependence among the uncertainties that affect distinct components of the objective function is present. Such a phenomenon cannot be ruled out in many relevant applications. For instance, in robust portfolio optimization the parameters influencing the revenue of a given portfolio also affect its risk. In this context, when we consider worst case scenarios, the minimum revenue occurrence does not usually coincide with the maximum risk situation. In this models the componentwise robust approach is unduly pessimistic, since the worst case scenario considered there is often unrealistic.

Several alternative notions of robust efficiency for uncertain vector problems have been defined (see, for example, the surveys [30] and [17]). In this work we will consider the approach introduced in [6] (see also [16]). Remarkably, the notions of robust efficiency in [6] are related to the so called upper type quasi order set relation (see, e.g., [25]) and can be considered in a deterministic set-valued optimization framework. Here the set minimization aproach gives relevance to worst case scenarios at each decision variable, taking into account possible interdependence among uncertainties. Necessary and sufficient robust optimality conditions can be obtained through scalarization, provided that suitable scalarizing functions $\Psi: 2^{Y} \rightarrow \mathbb{R} \cup\{ \pm \infty\}$ defined on a set-valued framework are given (key properties of scalarizing maps have been discussed, e.g., in [13]). The above described process, where scalarization is applied to the set-valued robust counterpart $R C-V P$ of the uncertain vector problem $V P(\mathcal{U})$, yields the deterministic scalar problem $S_{\Psi}-R C-V P$ (see Fig. 1).

Differently, when scalarization is applied directly to the original vector problem $V P(\mathcal{U})$ through a scalarizing function $\varphi: Y \rightarrow \mathbb{R} \cup\{ \pm \infty\}$, the uncertain scalar program $S_{\varphi}-V P(\mathcal{U})$ is obtained. Subsequently, the robust approach as introduced by [2] can be directly applied to $S_{\varphi}-V P(\mathcal{U})$ in order to obtain the deterministic scalar problem $R C-S_{\varphi}-V P$ (see Fig. 1). In this paper we investigate the relationship between $S_{\Psi}-R C-V P$ and $R C-S_{\varphi}-$ $V P$, where the scalarizing functions $\Psi$ and $\varphi$ are chosen independently from one another: under which conditions can scalarization and robustification processes be commuted? In detail, we study under which conditions a scalarization of the (set-valued) robust counterpart of the uncertain vector problem is equivalent to the robust counterpart of a scalarization of the same problem. The equivalence should be intended in the sense that both the two 
alternative approaches allow us to characterize the same set of robust optimal solutions of the original parametric problem $V P(\mathcal{U})$ by necessary and sufficient optimality conditions. In order to address this problem, on one hand, we note that general scalarization schemes to formulate problem $S_{\Psi}-R C-V P$ have been proposed in [13], where the authors identify key properties of scalarizing functions defined on set-valued frameworks that allow them to obtain necessary and sufficient robust optimality conditions through scalarization. On the other hand, we focus on the scalarization process used to formulate the uncertain scalar problem $S_{\varphi}-V P(\mathcal{U})$. To this purpose, we define a fairly general scalarization scheme that can be applied to the uncertain vector problem $V P(\mathcal{U})$. The classical robust approach developed in [3] can be directly applied on the scalarized uncertain problem $S_{\varphi}-V P(\mathcal{U})$ in order to obtain $R C-S_{\varphi}-V P$. Under appropriate assumptions on function $\varphi$, we prove that necessary and sufficient robust optimality conditions can be obtained. Therefore, problem $R C-S_{\varphi}-V P$ can be used as an alternative way to obtain necessary and sufficient robust optimality conditions. As a consequence, under this approach, all the known results on robust scalar optimization (see e.g. [2,3]) can be used in a consistent way with the robustness notions directly defined on the uncertain vector optimization problem. Hence, one can avoid the use of set-valued analysis tools without loosing the coherence with respect to the definitions of robustness notions defined in $[6,16]$. We underline that the use of optimality conditions obtained through scalarization on the set-valued robust counterpart $S_{\Psi}-R C-V P$ are, in a certain sense, less suitable for the use of practitioners. For instance, linear scalarizations, even if widely used in applications, are not well defined in a set-valued framework. Moreover, we remark that if linear scalarizations are directly applied on the uncertain vector program to formulate $R C-S_{\varphi}-V P$, robust optimality conditions that are not always coherent with the direct use of the robust approach in vector optimization are obtained. In Sect. 5, we analyze an example (see [4]) where there are robust efficient solutions of $V P(\mathcal{U})$ which cannot be obtained as solutions of the robust counterpart of the linearly scalarized problem $R C-S_{\varphi}-V P$. Nevertheless, all the robust efficient solutions of the same program can be found by scalarizing the set-valued robust counterpart $R C-V P$ with an appropriate nonlinear technique.

Remarkably, a commutativity problem, similar to the one we address here, has been studied in [8] for uncertain multiobjective programs under the componentwise robust approach. Furthermore, in [8], only the special setting of multiobjective optimization is considered with two particular scalarization methods. In this work, we consider a vector optimization problem with a generic ordering cone $K$ and a non componentwise approach to robustness (see [6]). In order to obtain a general result on commutativity of robustification and scalarization, we independently choose the scalarization processes $\Psi$ and $\varphi$ used in $S_{\Psi}-R C-V P$ and $R C-S_{\varphi}-V P$ in an abstract scalarization framework. In this context, the recent contribution presented in [11] deserves to be mentioned, where robustification and scalarization approaches have been considered in a componentwise setting and applied to the min-max regret approach.

The paper is organized as follows. In Sect. 2 we introduce notations and the properties that ensure necessary and sufficient optimality conditions through scalarization, along the line marked by [13]. In Sect. 3 we focus on two notions of robust efficiency and we highlight their connections with a set optimization approach. Then, we formulate problem $S_{\Psi}-R C-V P$ and we provide necessary and sufficient robust optimality conditions through scalarization. In Sect. 4, we formulate the robust counterpart of the uncertain scalarized vector program $R C-S_{\varphi}-V P$ and the requirements on the scalarizing functions $\varphi$ that ensure necessary and sufficient robust optimality conditions. In Sect. 5, we highlight the relations between the problems obtained following two alternative routes, where scalarization and robustification 
are switched (see Fig. 1). In Sect. 6, two applications using the Gerstewitz and the oriented distance approach to scalarization are considered in our framework. Such functions are widely used in scalarizations of vector optimization problems (see, e.g., [29] or [20] and the references therein). Moreover, in the special case of multiobjective optimization, several well known scalarization methods, such as Pascoletti-Serafini, $\epsilon$-constraint or Chebyshev scalarization, can be reduced to the use of the Gerstewitz scalarizing function (see e.g. Sect. 2.5 in [7]).

\section{Preliminary notions}

When not differently specified, $Y$ denotes a topological linear space and $2^{Y}$ denotes the collection of all subsets of $Y$. Given the set $A \in 2^{Y}, \partial A, \operatorname{cl} A$ and int $A$ denote, respectively, the boundary, the closure and the interior of $A$. The subset $K \subseteq Y$ will denote a closed and convex cone. The cone $K$ is said to be pointed when $K \cap(-K)=\{0\}$ and solid when int $K \neq \emptyset$. The partial order structure induced on $Y$ by the cone $K$ is:

$$
y, y^{\prime} \in Y, y^{\prime} \leq y \Longleftrightarrow y-y^{\prime} \in K
$$

The set $A-K$ denotes the algebraic difference between the sets $A$ and $K$. The set $A \subseteq Y$ is said to be $K$-proper and $K$-closed when respectively $A-K \neq Y$ and $\partial(A-K) \subseteq A-K$. The following results will be used in the sequel.

Lemma 2.1 Let $A \subseteq Y$ be $K$-proper. If $y \in \partial(A-K)$, then, for all $a \in \operatorname{cl}(A-K)$, either $y \not \leq a$ or $y-a \in \partial(-K)$ holds.

Proof We will prove that, if an element $a \in \operatorname{cl}(A-K)$ exists such that $y \leq a$, then the element $k \in K$ for which $y=a-k$ holds, is such that $k \in \partial K$. Clearly, whenever int $K=\emptyset$, $k \in \partial K$. If int $K \neq \emptyset$, let $k \in \operatorname{int} K$. Then, there exists a neighborhood $\mathcal{U}$ of 0 such that $\{k\}+\mathcal{U} \subseteq K$. Since $a \in A-K$, then $\{a-k\}+\mathcal{U} \subseteq A-K$. However, since $y=a-k$, it follows $\{a-k\}+\mathcal{U}=\{y\}+\mathcal{U} \subseteq A-K$. Hence, $y \notin \partial(A-K)$, a contradiction.

Lemma 2.2 Let $A \subseteq Y$ be $K$-proper and $K$-closed. Then, $A \cap \partial(A-K) \neq \emptyset$ holds.

Proof Since $\partial(A-K) \subseteq A-K$, then, for all $a^{\prime} \in \partial(A-K)$, there exists $a \in A$ and $k \in K$ such that

$$
a^{\prime}=a-k
$$

We claim that $a \in \partial(A-K)$. Indeed, suppose by contradiction that $a \notin \partial(A-K)$. This implies $a \in \operatorname{int}(A-K)$. Hence, a neighborhood $\mathcal{U}$ of 0 exists such that $\{a\}+\mathcal{U} \subseteq A-K$ holds. Moreover, since $k \in K$, it follows $\{a-k\}+\mathcal{U} \subseteq A-K$, namely $a^{\prime}=a-k \in \operatorname{int}(A-K)$, a contradiction.

We consider the partial quasi order induced on $2^{Y}$ by the ordering cone $K$ through the so called upper type relation (see [25] and [26]).

Definition 2.3 Let $A, B \in 2^{Y}$. Then $B \prec A \Longleftrightarrow B \subseteq A-K$.

Given $A, B \in 2^{Y}$, the relations $\sim$ and $\prec$ are defined as follows

$$
\begin{aligned}
& B \sim A \quad \Longleftrightarrow A \prec B \text { and } B \prec A \\
& B \prec A \quad \Longleftrightarrow B \prec A \text { and } A \text { ₹ } B
\end{aligned}
$$


When sets are singletons, relation $\prec$ reduces to $\leq$ : for $\{y\},\left\{y^{\prime}\right\} \in 2^{Y}$, the relation $\{y\} \prec\left\{y^{\prime}\right\}$ is equivalent to $y \leq y^{\prime}$.

We retrieve here the notions of (strictly) そ-preserving and (strictly) そ-representing scalarizing functions (considered e.g. in [13] and more recently in [12]) and we refer them to the quasi order relation $\prec$. We note that the order preserving properties of a function are closely related to monotonicity, while the order representing properties compare level sets of the function with the order structure of the underlying space. We remark that, when an optimization problem is tackled through scalarization, the order preserving (resp. representing) properties are essential to obtain sufficient (resp. necessary) optimality conditions through scalarization.

Definition 2.4 Let $\mathcal{A} \subseteq 2^{Y}$ be a collection of sets and $A \in \mathcal{A}$. A function $\Psi: \mathcal{A} \rightarrow \mathbb{R} \cup\{ \pm \infty\}$ is said to be:

a) そ-preserving at $A$ on $\mathcal{A}$ if

$$
B \in \mathcal{A}, B \prec A \Longrightarrow \Psi(B) \leq \Psi(A)
$$

b) strictly $\prec$-preserving at $A$ on $\mathcal{A}$ if

$$
B \in \mathcal{A}, B \prec A \Longrightarrow \Psi(B)<\Psi(A)
$$

Definition 2.5 Let $\mathcal{A} \subseteq 2^{Y}$ be a collection of sets and $A \in \mathcal{A}$. A function $\Psi: \mathcal{A} \rightarrow \mathbb{R} \cup\{ \pm \infty\}$ is said to be

a) そ-representing at $A$ on $\mathcal{A}$ when

$$
B \in \mathcal{A}, \Psi(B)<\Psi(A) \Longrightarrow B \prec A
$$

b) strictly $\prec$-representing at $A$ on $\mathcal{A}$ when

$$
B \in \mathcal{A}, \Psi(B) \leq \Psi(A) \Longrightarrow B \prec A
$$

We refer the reader to $[13,14,18,19]$, among others, for contributions where several functions defined on set-valued frameworks with the above mentioned order preserving and order representing properties have been considered.

Remark 2.6 We will consider the restriction of property $a$ ) in Definition 2.4 to the vector case where, when sets are singletons, the set relation $\prec$ reduces to $\leq$. Given $A \subseteq Y$, a function $\psi: Y \rightarrow \mathbb{R}$ is said to be $\leq$-preserving at $a \in A$ on $A$ when

$$
b \in A, b \leq a \Longrightarrow \psi(b) \leq \psi(a)
$$

In addition, we will consider the restriction of property $b$ ) in Definition 2.5 to the vector case. Given $A \subseteq Y$, a function $\psi: Y \rightarrow \mathbb{R}$ is said to be strictly $\leq$-representing at $a \in A$ on $A$ when

$$
b \in A, \psi(b) \leq \psi(a) \Longrightarrow b \leq a
$$

The following Example shows that monotone increasing linear functions are everywhere $\leq$-preserving and nowhere $\leq$-representing on $\mathbb{R}^{2}$.

Example 2.7 Let $\mathbb{R}^{2}$ be endowed with the partial quasi order induced by the cone $K=\mathbb{R}_{+}^{2}$ and let the linear function $\Lambda: \mathbb{R}^{2} \rightarrow \mathbb{R}$ be such that $\Lambda\left(x_{1}, x_{2}\right)=\lambda x_{1}+(1-\lambda) x_{2}$ with 
$\lambda \in[0,1]$. The function $\Lambda$ is $\leq$-preserving at any $y$ on $\mathbb{R}^{2}$. Indeed, given two elements $y, y^{\prime} \in \mathbb{R}^{2}$, the relation $\Lambda\left(y^{\prime}\right) \leq \Lambda(y)$ is equivalent to

$$
\lambda\left(y_{1}^{\prime}-y_{1}\right)+(1-\lambda)\left(y_{2}^{\prime}-y_{2}\right) \leq 0
$$

which, in turn, is satisfied whenever $y_{1}^{\prime} \leq y_{1}$ and $y_{2}^{\prime} \leq y_{2}$, namely $y^{\prime} \leq y$. Differently, the function $\Lambda$ is not $\leq$-representing at any $y$ on $\mathbb{R}^{2}$. Indeed, for any $y$, an element $y^{\prime}$ can be found such that the relation $\Lambda\left(y^{\prime}\right) \leq \Lambda(y)$ does not imply $y^{\prime} \leq y$. For example, if $\lambda \neq 0$, pick

$$
y^{\prime}=\left(y_{1}-\frac{1-\lambda+\epsilon}{\lambda}, y_{2}+1\right)
$$

and $y^{\prime}=\left(y_{1}+\epsilon, y_{2}-\epsilon\right)$ if $\lambda=0$, where $\epsilon>0$. Clearly, $y^{\prime} \not \leq y$ always holds. However, in both cases, it results $\Lambda\left(y^{\prime}\right)=\Lambda(y)-\epsilon \leq \Lambda(y)$.

Remark 2.8 In Sect. 6, we will consider two non linear functions on vector spaces (the so called Gerstewitz and oriented distance functions) whose relevant properties in the context of non linear separation and scalarization have been respectively deepened in [9] and [31]. Provided that appropriate parameters' selections are performed, both such functions satisfy the $\leq$-preserving and strictly $\leq$-preserving properties.

Let us denote by $\mathcal{X}$ the decision space and let $f: \mathcal{X} \times \mathcal{U} \longrightarrow Y$ be a vector valued parameterized objective function, where $\mathcal{U}$ is the set of possible parameters' realizations. The family $V P(\mathcal{U})$ of parametric vector optimization problems is

$$
\text { minimize } f(x, \xi) \text { subject to } x \in \mathcal{X} \quad V P(\mathcal{U})
$$

where $\mathcal{X} \subseteq X$ is the feasible set and $\xi \in \mathcal{U}$.

We recall the notions of robust strict efficiency and robust efficiency in vector optimization (see $[6,16])$.

Definition 2.9 The element $x_{0} \in \mathcal{X}$ is said to be robust strictly efficient when there exists no $x \in \mathcal{X} \backslash\left\{x_{0}\right\}$ such that

$$
\forall \xi \in \mathcal{U}, \exists \xi^{\prime} \in \mathcal{U}: f(x, \xi) \leq f\left(x_{0}, \xi^{\prime}\right)
$$

The element $x_{0} \in \mathcal{X}$ is said to be robust efficient when there is no $x \in \mathcal{X} \backslash\left\{x_{0}\right\}$ such that

$$
\forall \xi \in \mathcal{U} \exists \xi^{\prime} \in \mathcal{U}: f(x, \xi) \leq f\left(x_{0}, \xi^{\prime}\right)
$$

and

$$
\exists \bar{\xi} \in \mathcal{U} \text { s.t. } f\left(x_{0}, \bar{\xi}\right) \not \leq f(x, \xi), \forall \xi \in \mathcal{U}
$$

Remark 2.10 The above notions of robust (strict) efficiency have been considered in [6] in the multiobjective case, where $Y=\mathbb{R}^{p}$ and $K=\mathbb{R}_{+}^{p}$, and extended in [16] to vector optimization problems with general ordering cones. In a certainty setting, where the set of parameters $\mathcal{U}$ is a singleton, the notion of robust efficiency reduces to the usual notion of efficiency in vector optimization. Moreover, in vector optimization the robust strict efficiency definition reduces to a strict efficiency notion that coincides with efficiency, whenever the vector objective function $f$ is injective at the solutions and the ordering cone $K$ is pointed.

We will denote by $\mathcal{F} \subseteq 2^{Y}$ the collection of sets

$$
\mathcal{F}:=\left\{\bigcup_{\xi \in \mathcal{U}} f(x, \xi) \subseteq Y: x \in \mathcal{X}\right\}
$$




\section{Parametric vector optimization: scalarization of the robust counterpart}

In this Section we refer to the approach described on the left hand side of Fig. 1. First, we introduce the set-valued robust counterpart of a parametric vector optimization problem. Subsequently, we consider a suitable scalarization that yields the deterministic scalar problem $S_{\Psi}-R C-V P$. Using some scalarization results in [13], we obtain necessary and sufficient robust optimality conditions on the original uncertain vector problem.

In order to formulate the (set-valued) robust counterpart of the uncertain vector optimization problem $V P(\mathcal{U})$ introduced in Sect. 2, let us introduce the map of achievable objective values $F: \mathcal{X} \rightarrow 2^{Y}$ defined as

$$
F(x):=\{f(x, \xi): \xi \in \mathcal{U}\} \in \mathcal{F}, \quad \forall x \in \mathcal{X}
$$

We reformulate the definitions of robust efficiency on the set-valued objective map $F$ by means of the upper type quasi order "२”" induced on $2^{Y}$ by the ordering cone $K$ (see Definition 2.3). It is easy to see that the element $x_{0} \in \mathcal{X}$ is robust strictly efficient if and only if

$$
\forall x \in \mathcal{X} \backslash\left\{x_{0}\right\}, F(x) \nprec F\left(x_{0}\right)
$$

Moreover, the element $x_{0} \in \mathcal{X}$ is robust efficient if and only if

$$
x \in \mathcal{X}, F(x) \prec F\left(x_{0}\right) \Longrightarrow F\left(x_{0}\right) \prec F(x)
$$

Hence, the notions of robust efficiency introduced in Definition 2.9 are consistent with a set optimization framework. The minimization of the set-valued objective $F: \mathcal{X} \rightarrow 2^{Y}$ with respect to the upper quasi order $\prec$ yelds a deterministic set-valued program that can be interpreted as the (set-valued) robust counterpart of the uncertain vector problem $V P(\mathcal{U})$ :

$$
\prec-\text { minimize } F(x) \text { subject to } x \in \mathcal{X} \quad R C-V P
$$

We provide some robust optimality conditions obtained by scalarizing the deterministic setvalued optimization problem $R C-V P$. Let $\Psi: 2^{Y} \rightarrow \mathbb{R} \cup\{ \pm \infty\}$. We consider the following scalar problem, obtained from the set-valued robust counterpart $R C-V P$ by scalarization through $\Psi$ :

$$
\text { minimize } \Psi(F(x)) \text { subject to } x \in \mathcal{X} \quad S_{\Psi}-R C-V P
$$

\subsection{Robust optimality conditions through $S_{\Psi}-R C-V P$}

We consider the axiomatic approach to scalarization in set optimization introduced in [13]. This approach provides necessary and sufficient optimality conditions whenever the scalarizing function is characterized by (strictly) そ-preserving and (strictly) そ-representing properties. Moreover, we show that such properties cannot be omitted in order to obtain necessary and sufficient optimality conditions. Hence, all the scalarization methods in set optimization that provide necessary and sufficient optimality conditions can be framed within this scheme.

\section{Robust strict efficiency}

The following Proposition shows the relation between the $\prec$-preserving property of $\Psi$ and sufficient robust strict optimality conditions through scalarization. 
Proposition 3.1 Let $\Psi$ be $\prec$-preserving at $F\left(x_{0}\right)$ on $\mathcal{F}$. If $x_{0}$ is the unique solution of $S_{\Psi}-$ $R C-V P$, then it is robust strictly efficient. Conversely, if $x_{0}$ is robust strictly efficient whenever it is the unique solution of $S_{\Psi}-R C-V P$, then $\Psi$ is $\prec$-preserving at $F\left(x_{0}\right)$ on $\mathcal{F}$.

Proof See Proposition 3.10 in [13] for the first implication. Conversely, we need to prove that the implication

$$
\Psi(F(x))>\Psi\left(F\left(x_{0}\right)\right), \forall x \in \mathcal{X} \backslash\left\{x_{0}\right\} \Longrightarrow F(x) \nprec F\left(x_{0}\right), \forall x \in \mathcal{X} \backslash\left\{x_{0}\right\}
$$

entails that $\Psi$ is $\prec$-preserving at $F\left(x_{0}\right)$ on $\mathcal{F}$. By contradiction, suppose that there exists $\bar{x} \in \mathcal{X} \backslash\left\{x_{0}\right\}$ such that $F(\bar{x}) \prec F\left(x_{0}\right)$ and $\Psi(F(\bar{x}))>\Psi\left(F\left(x_{0}\right)\right)$. Hence, the occurrence $\Psi(F(x))>\Psi\left(F\left(x_{0}\right)\right), \forall x \in \mathcal{X} \backslash\left\{x_{0}\right\}$, does not imply $F(x) \nprec F\left(x_{0}\right)$, for all $x \in \mathcal{X} \backslash\left\{x_{0}\right\}$, a contradiction.

The following Proposition shows the relation between the strict $\prec$-representing property of $\Psi$ and necessary robust strict optimality conditions through scalarization.

Proposition 3.2 Let $\Psi$ be strictly $\prec$-representing at $F\left(x_{0}\right)$ on $\mathcal{F}$. If the element $x_{0} \in \mathcal{X}$ is robust strictly efficient, then it is the unique solution of problem $S_{\Psi}-R C-V P$. Conversely, if $x_{0}$ is the unique solution of $S_{\Psi}-R C-V P$ whenever it is robust strictly efficient, then $\Psi$ is strictly $\prec$-representing at $F\left(x_{0}\right)$ on $\mathcal{F}$.

Proof See Proposition 3.6 in [13] for the first implication. Conversely, we need to prove that the implication

$$
F(x) \not F\left(x_{0}\right), \forall x \in \mathcal{X} \backslash\left\{x_{0}\right\} \Longrightarrow \Psi(F(x))>\Psi\left(F\left(x_{0}\right)\right), \forall x \in \mathcal{X} \backslash\left\{x_{0}\right\}
$$

entails that $\Psi$ is strictly $\prec$-representing at $F\left(x_{0}\right)$ on $\mathcal{F}$. By contradiction, suppose that there exists $\bar{x} \in \mathcal{X} \backslash\left\{x_{0}\right\}$ such that $F(\bar{x}) \nprec F\left(x_{0}\right)$ and $\Psi(F(\bar{x})) \leq \Psi\left(F\left(x_{0}\right)\right)$. Hence, the occurrence $F(x) \nprec F\left(x_{0}\right)$ for all $x \in \mathcal{X} \backslash\left\{x_{0}\right\}$ does not imply $\Psi(F(x))>\Psi\left(F\left(x_{0}\right)\right)$, for all $x \in \mathcal{X} \backslash\left\{x_{0}\right\}$, a contradiction.

\section{Robust efficiency}

The following Proposition shows the relation between the strict $\prec$-preserving property of $\Psi$ and sufficient robust optimality conditions through scalarization.

Proposition 3.3 Let $\Psi$ be strictly $\prec$-preserving at $F\left(x_{0}\right)$ on $\mathcal{F}$. If $x_{0} \in \mathcal{X}$ is a solution of problem $S_{\Psi}-R C-V P$, then it is robust efficient. Conversely, if $x_{0} \in \mathcal{X}$ is robust efficient whenever it is a solution of problem $S_{\Psi}-R C-V P$, then $\Psi$ is strictly $\prec-p r e s e r v i n g$ at $F\left(x_{0}\right)$ on $\mathcal{F}$.

Proof See Proposition 3.9 in [13] for the first implication. Conversely, we need to prove that the implication

$$
\Psi(F(x)) \geq \Psi\left(F\left(x_{0}\right)\right), \forall x \in \mathcal{X} \Longrightarrow F(x) \nprec F\left(x_{0}\right) \text { or } F(x) \sim F\left(x_{0}\right), \forall x \in \mathcal{X}
$$

entails that $\Psi$ is strictly $\prec$-preserving at $F\left(x_{0}\right)$ on $\mathcal{F}$. By contradiction, suppose that there exists $\bar{x} \in \mathcal{X} \backslash\left\{x_{0}\right\}$ such that $F(\bar{x}) \prec F\left(x_{0}\right)$, with $F(\bar{x}) \nsim F\left(x_{0}\right)$ and $\Psi(F(\bar{x})) \geq \Psi\left(F\left(x_{0}\right)\right)$. Hence, the occurrence $\Psi(F(x)) \geq \Psi\left(F\left(x_{0}\right)\right)$, for all $x \in \mathcal{X}$, does not imply either $F(x) \nprec F\left(x_{0}\right)$ or $F(x) \sim F\left(x_{0}\right)$, for all $x \in \mathcal{X}$, a contradiction. 
The following Proposition shows the relation between the $\prec$-representing property of $\Psi$ and necessary robust optimality conditions through scalarization.

Proposition 3.4 Let $\Psi$ be $\prec$-representing at $F\left(x_{0}\right)$ on $\mathcal{F}$. If $x_{0} \in \mathcal{X}$ is robust efficient then it is a solution of problem $S_{\Psi}-R C-V P$. Conversely, if $x_{0} \in \mathcal{X}$ is a solution of problem $S_{\Psi}-R C-V P$ whenever it is robust efficient, then $\Psi$ is $\prec$-representing at $F\left(x_{0}\right)$ on $\mathcal{F}$.

Proof See Proposition 3.5 in [13] for the first implication. Conversely, we need to prove that the implication

$$
F(x) \nprec F\left(x_{0}\right) \text { or } F(x) \sim F\left(x_{0}\right), \forall x \in \mathcal{X} \Longrightarrow \Psi(F(x)) \geq \Psi\left(F\left(x_{0}\right)\right), \forall x \in \mathcal{X}
$$

entails that $\Psi$ is $\prec$-representing at $F\left(x_{0}\right) \in \mathcal{F}$ on $\mathcal{F}$. By contradiction, suppose that there exists $\bar{x} \in \mathcal{X} \backslash\left\{x_{0}\right\}$ such that $\Psi(F(\bar{x}))<\Psi\left(F\left(x_{0}\right)\right)$ and $F(\bar{x}) \nprec F\left(x_{0}\right)$ or $F(\bar{x}) \sim F\left(x_{0}\right)$. Hence, $F(x) \nprec F\left(x_{0}\right)$ or $F(x) \sim F\left(x_{0}\right)$, for all $x \in \mathcal{X}$, do not imply $\Psi(F(x)) \geq \Psi\left(F\left(x_{0}\right)\right)$ for all $x \in \mathcal{X}$, a contradiction.

\subsection{Comparison between the set-valued robust and the componentwise robust approaches}

The robust approach as introduced by [2] for scalar uncertain programs, has been extended to uncertain vector optimization problems in different ways (see, e.g., the surveys [30] and [17]). The set-valued robust approach gives relevance to the worst possible realizations of uncertain parameters at each decision variable through the upper quasi order "२”". Differently, the so called componentwise robust approach, introduced in [27] and widely considered in applications in the special case of uncertain multiobjective problems (see e.g. [10] or [8] and the reference therein), considers the worst case at each decision variable to be a single point, whose coordinates match the componentwise worst cases achieved by each component of the uncertain objective. Let $Y=\mathbb{R}^{p}$ and $K=\mathbb{R}_{+}^{p}$, the componentwise worst case is defined as the multiobjective function $\mu: \mathcal{X} \rightarrow \mathbb{R}^{p}$

$$
\mu(x)=\left(\begin{array}{cc}
\max _{\xi \in \mathcal{U}} & f_{1}(x, \xi) \\
\vdots \\
\max _{\xi \in \mathcal{U}} & f_{p}(x, \xi)
\end{array}\right), \forall x \in \mathcal{X}
$$

Note that, in case of conflicting objectives, the componentwise worst case $\mu(x)$ may not be an element of $F(x)$, the set of achievable objective values at $x$.

The componentwise robust counterpart of $V P(\mathcal{U})$ is

$$
\text { minimize } \mu(x) \text { subject to } x \in \mathcal{X}
$$

Some interesting results on commutativity between robustification and scalarization were proved in [8] (see Proposition 3.6) for linear scalarization and $\epsilon$-constraint scalarization. We underline that, with the componentwise approach, the robust counterpart is a vector-valued function as in the original uncertain vector optimization problem. In the special case of uncertain multiobjective problems, where $Y=\mathbb{R}^{p}$ and $K=\mathbb{R}_{+}^{p}$, if, for all $x \in \mathcal{X}$, the relation $\mu(x) \in F(x)$ holds, i.e. $F(x)-K=\{\mu(x)\}-K$, the set-valued worst case scenario at each decision variable is attained at a unique point. In this event, the robust solutions considered in $R C-V P$ coincide with the solutions of the componentwise robust counterpart. Whenever, for some $x \in \mathcal{X}$, there exists no element $y \in F(x)$ such that $F(x)-K=\{y\}-K$, the componentwise robust approach is unduly pessimistic. This typically happens, for instance, in 
mean-variance portfolio optimization problems (see e.g. the case of elliptic joint uncertainty set considered in [8]). Moreover, the set-valued robust approach allows us to discard some dominated situations that cannot be distinguished by using the componentwise approach. This is shown in the following example, where an uncertain vector problem is tackled through both the approaches.

Example 3.5 Let $Y=\mathbb{R}^{2}, K=\mathbb{R}_{+}^{2}, \mathcal{X}=[-1,0]$ and $\mathcal{U}=[0,1]$. We consider the following uncertain vector problem

$$
\text { minimize } f(x, \xi) \quad \text { s.t. } \quad x \in[-1,0] \quad V P_{E x(3.5)}(\mathcal{U})
$$

where $f: \mathcal{X} \times \mathcal{U} \rightarrow \mathbb{R}^{2}$ is defined by

$$
f(x, \xi)=\left(\begin{array}{c}
\xi x \\
(1-\xi) x
\end{array}\right) ; x \in[-1,0], \xi \in[0,1]
$$

The robustification of problem $V P_{E x(3.5)}(\mathcal{U})$ through the set-valued approach leads to the formulation of the following set-valued program

$$
\prec-\operatorname{minimize} F(x) \quad \text { s.t. } \quad x \in[-1,0]
$$

where the set-valued map $F: \mathcal{X} \rightarrow 2^{\mathbb{R}^{2}}$ is

$$
F(x)=\bigcup_{\xi \in[0,1]}\left(\begin{array}{c}
\xi x \\
(1-\xi) x
\end{array}\right)=\operatorname{conv}\left\{\left(\begin{array}{l}
x \\
0
\end{array}\right),\left(\begin{array}{l}
0 \\
x
\end{array}\right)\right\} ; x \in[-1,0]
$$

and where conv $\{a, b\}$ is the convex hull of $\{a, b\}$. Clearly, according to Definition $2.9, x=-1$ is the unique robust efficient solution of $V P(\mathcal{U})$. In Fig. 2, a representation of some set values of map $F$ is provided. However, the vector function $\mu: \mathcal{X} \rightarrow \mathbb{R}^{2}$ is

$$
\mu(x)=\left(\begin{array}{lc}
\max _{\xi \in[0,1]} & \xi x \\
\max _{\xi \in[0,1]}(1-\xi) x
\end{array}\right)=\left(\begin{array}{l}
0 \\
0
\end{array}\right) ; x \in[-1,0]
$$

Since $\mu$ is constant, any $x \in[-1,0]$ is a componentwise robust solution of problem $V P_{E x(3.5)}(\mathcal{U})$.

\section{Parametric vector optimization: the robust counterpart of the scalarized problem}

In this Section, we refer to the approach $R C-S_{\varphi}-V P$ as described on the right hand side of Fig. 1. With this approach, a scalarization of the uncertain vector optimization problem is considered in $S_{\varphi}-V P(\mathcal{U})$. Under appropriate conditions, we will show that its classical robust counterpart $R C-S_{\varphi}-V P$ allows us to obtain necessary and sufficient robust optimality conditions.

\subsection{A general scalarization scheme}

We introduce an axiomatic approach to scalarization which allows us to identify a class of scalarizing functions that are suitable for our purpose. 
Fig. 2 Set values of map $F$ at $x=-1, x=0$ and generic $x \in(-1,0)$

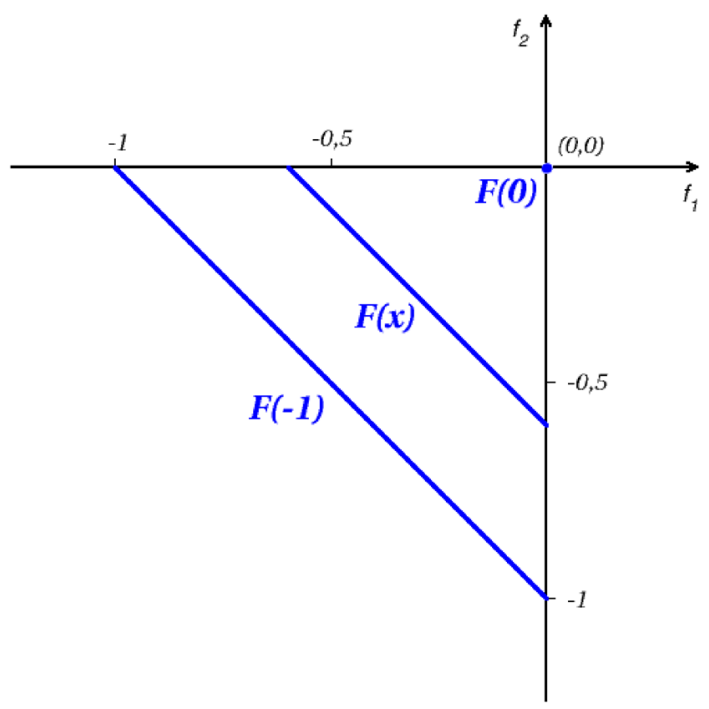

Let $A \in 2^{Y}$ be nonempty and let $\varphi: Y \rightarrow \mathbb{R} \cup\{ \pm \infty\}$. Let us introduce a scalarizing function $\varphi_{A-K}: Y \rightarrow \mathbb{R} \cup\{ \pm \infty\}$ as follows:

$$
\varphi_{A-K}(y):=\inf _{a \in A-K} \varphi(y-a), \forall y \in Y
$$

The family of uncertain scalar programs obtained from $V P(\mathcal{U})$ by scalarization through $\varphi_{A-K}$ is

$$
\text { minimize } \varphi_{A-K}(f(x, \xi)) \text { subject to } x \in \mathcal{X} \quad S_{\varphi}-V P(\mathcal{U})
$$

The robust approach introduced in [3] can be directly applied to $S_{\varphi}-V P(\mathcal{U})$. The worst case scenario at the decision variable $x \in \mathcal{X}$ is

$$
\sup _{\xi \in \mathcal{U}} \varphi_{A-K}(f(x, \xi))
$$

Let the map $\Phi_{A-K}: \mathcal{F} \rightarrow \mathbb{R} \cup\{ \pm \infty\}$ be defined by

$$
\Phi_{A-K}(C):=\sup _{c \in C} \varphi_{A-K}(c), \quad \forall C \in \mathcal{F}
$$

The worst case scenario at $x \in \mathcal{X}$ can be expressed in terms of $\Phi_{A-K}$ as follows

$$
\sup _{\xi \in \mathcal{U}} \varphi_{A-K}(f(x, \xi))=\sup _{y \in F(x)} \varphi_{A-K}(y)=\Phi_{A-K}(F(x))
$$

Then, the robust counterpart of the scalar uncertain problem $S_{\varphi}-V P(\mathcal{U})$ results

$$
\text { minimize } \Phi_{A-K}(F(x)) \text { subject to } x \in \mathcal{X} \quad R C-S_{\varphi}-V P
$$

We will show that necessary and sufficient robust optimality conditions can be obtained through scalarization of $V P(\mathcal{U})$ by means of the function $\varphi_{A-K}$, provided that suitable conditions on $\varphi$ are given. 


\subsubsection{Properties of problem $R C-S_{\varphi}-V P$}

In this subsection we prove that, under appropriate conditions, the function $\Phi_{A-K}$ that represents the robust counterpart of the scalarization of the uncertain vector optimization problem inherits some fundamental properties from the original scalarizing function $\varphi$.

To this purpose, we will provide sufficient conditions for some properties of the function $\varphi_{A-K}$ that will be useful in the sequel. Besides the $\leq$-preserving and strictly $\leq$-representing properties of $\varphi$ at 0 on $Y$, we will exploit the following property of $\varphi$ :

$$
y \in \partial(-K) \Longrightarrow \varphi(y)=\varphi(0)
$$

that relates the level set of $\varphi$ at 0 with the order structure.

Remark 4.1 The restriction of property b) in Definition 2.4 to the vector case entails that a function $\varphi$ is strictly $\leq$-preserving at 0 on $Y$ when

$$
y \in Y, y \leq 0 \text { and } y \neq 0 \Longrightarrow \varphi(y)<\varphi(0)
$$

Here we remark that $\varphi$ cannot be strictly $\leq$-preserving at 0 on $Y$ if property $(\mathrm{P})$ holds. Indeed, for any $y \in \partial(-K)$ such that $y \neq 0$, property $(\mathrm{P})$ implies $\varphi(y)=\varphi(0)$.

Moreover, the following assumption on the nonempty set $A \subseteq Y$ will be considered.

Assumption 4.2 The infimum

$$
\varphi_{A-K}(y)=\inf _{a \in A-K} \varphi(y-a)
$$

is attained for all $y \notin A-K$.

Remark 4.3 Similar attainment assumptions, involving both the function $\varphi$ and the set $A-K$, have been considered in the context of robust multiobjective optimization (see, e.g., [1]), in robust vector optimization frameworks (see, e.g., [21]) and in order to provide characterizations of set relations via scalarization in [24] and [5].

Lemma 4.4 Let $A \subseteq Y$ be nonempty.

1) If $\varphi$ is $\leq$-preserving at 0 on $Y$, then

$$
y \in A-K \Longrightarrow \varphi_{A-K}(y) \leq \varphi(0)
$$

2) If $\varphi$ is $\leq$-preserving and strictly $\leq$-representing at 0 on $Y$ and $(P)$ holds, then

$$
y \in \partial(A-K) \Longrightarrow \varphi_{A-K}(y)=\varphi(0)
$$

whenever $A$ is $K$-proper and $K$-closed.

3) If $\varphi$ is $\leq$-preserving and strictly $\leq$-representing at 0 on $Y$ and $(P)$ holds, then

$$
\varphi_{A-K}(y) \leq \varphi(0) \Longrightarrow y \in A-K
$$

whenever Assumption 4.2 is fulfilled for $\varphi$ on A.

Proof 1) If $y \in A-K$, there exists $a \in A$ such that $y-a \leq 0$, which implies

$$
\varphi(y-a) \leq \varphi(0)
$$


since $\varphi$ is $\leq$-preserving at 0 . Hence,

$$
\varphi_{A-K}(y)=\inf _{a^{\prime} \in A-K} \varphi\left(y-a^{\prime}\right) \leq \varphi(y-a) \leq \varphi(0)
$$

and the implication is proved.

2) If $y \in \partial(A-K)$, then either $y-a \not \leq 0$ or $y-a \in \partial(-K)$ holds, for all $a \in A-K$ (see Lemma 2.1). If $y-a \nless \leq 0$, then $\varphi(y-a)>\varphi(0)$, since $\varphi$ is strictly $\leq$-representing at 0 on $Y$. If $y-a \in \partial(-K)$, then $\varphi(y-a)=\varphi(0)$, by (P). Hence, $\varphi(y-a) \geq \varphi(0)$, for all $a \in A-K$. Therefore,

$$
\varphi_{A-K}(y) \geq \varphi(0)
$$

Moreover, since $A$ is $K$-closed, $y \in A-K$ holds; by 1$)$ it follows that $\varphi_{A-K}(y) \leq \varphi(0)$. To conclude, $\varphi_{A-K}(y)=\varphi(0)$.

3) By contradiction, suppose that $\varphi_{A-K}(y) \leq \varphi(0)$ and $y \notin A-K$. Since $y-a \not \leq 0$ for all $a \in A-K$, then

$$
\varphi(y-a)>\varphi(0), \quad \forall a \in A-K
$$

since $\varphi$ is strictly $\leq$-representing at 0 on $Y$. Hence, $\inf _{a \in A-K} \varphi(y-a)=\varphi_{A-K}(y) \geq \varphi(0)$. Then

$$
\varphi_{A-K}(y)=\varphi(0)
$$

Relations (4) and (5) imply that the infimum inf $\operatorname{i\in A-K} \varphi(y-a)=\varphi_{A-K}(y)$ is not attained in $A-K$. This leads to a contradiction, under Assumption 4.2.

Lemma 4.5 Let $A \subseteq Y$ be nonempty. Then $\Phi_{A-K}(A) \leq \varphi(0)$. Moreover, if $A$ is $K$-proper and $K$-closed, $\varphi$ is $\leq$-preserving and strictly $\leq$-representing at 0 on $Y$ and property $(P)$ holds, then $\Phi_{A-K}(A)=\varphi(0)$.

Proof For any given element $a^{\prime} \in A$, the relation

$$
\varphi_{A}\left(a^{\prime}\right)=\inf _{a \in A} \varphi\left(a^{\prime}-a\right) \leq \varphi(0)
$$

holds. Moreover, it follows

$$
\varphi_{A-K}\left(a^{\prime}\right) \leq \varphi_{A}\left(a^{\prime}\right) \leq \varphi(0)
$$

Hence

$$
\Phi_{A-K}(A)=\sup _{a^{\prime} \in A} \varphi_{A-K}\left(a^{\prime}\right) \leq \varphi(0)
$$

Now, let $A$ be $K$-proper and $K$-closed. For any element $a^{\prime} \in A$, it results

$$
\varphi_{A-K}\left(a^{\prime}\right) \leq \sup _{a^{\prime} \in A} \varphi_{A-K}\left(a^{\prime}\right)=\Phi_{A-K}(A)
$$

Taking into account Lemma 2.2, an element $\bar{a} \in A \cap \partial(A-K)$ can be chosen and the previous relation together with implication 2) leads to

$$
\varphi(0)=\varphi_{A-K}(\bar{a}) \leq \Phi_{A-K}(A)
$$

The thesis follows from (6) and (7).

The following Lemma provides some sufficient conditions for $\Phi_{A-K}$ to be $\prec$-preserving at $A$ on $2^{Y}$. 
Lemma 4.6 Let $A \subseteq Y$ be nonempty, $K$-proper and $K$-closed. If $\varphi$ is $\leq$-preserving and strictly $\leq$-representing at 0 on $Y$ and property $(P)$ holds, then the map $\Phi_{A-K}$ is $\prec$-preserving at $A$ on $2^{Y}$.

Proof Let $A_{1} \in 2^{Y}$ be such that $A_{1} \prec A$. Then, for all $a_{1} \in A_{1}$, it holds $a_{1} \in A-K$. By 1 ) in Lemma 4.4, it implies

$$
\varphi_{A-K}\left(a_{1}\right) \leq \varphi(0)
$$

for all $a_{1} \in A_{1}$. Hence, taking into account Lemma 4.5, it follows

$$
\Phi_{A-K}\left(A_{1}\right) \leq \varphi(0)=\Phi_{A-K}(A)
$$

which proves the thesis.

The following Lemma provides some sufficient conditions for $\Phi_{A-K}$ to be strictly $\prec-$ representing at $A$ on $2^{Y}$.

Lemma 4.7 Let $A \subseteq Y$ be nonempty and let Assumption 4.2 hold for $\varphi$ on A. If $\varphi$ is $\leq-$ preserving and strictly $\leq-$ representing at 0 on $Y$ and $(P)$ holds, then the map $\Phi_{A-K}$ is strictly $\prec$-representing at $A$ on $2^{Y}$.

Proof Let $A_{1} \in 2^{Y}$ and let

$$
\Phi_{A-K}\left(A_{1}\right) \leq \Phi_{A-K}(A)
$$

By Lemma 4.5), $\sup _{a_{1} \in A_{1}} \varphi_{A-K}\left(a_{1}\right) \leq \Phi_{A-K}(A)=\varphi(0)$. Hence, for all $a_{1} \in A_{1}$, it holds

$$
\varphi_{A-K}\left(a_{1}\right) \leq \varphi(0)
$$

By 3 ) in Lemma 4.4, it holds $a_{1} \in A-K$, for all $a_{1} \in A_{1}$, namely $A_{1} \prec A$. The thesis is proved.

\subsubsection{Robust optimality conditions through $R C-S_{\varphi}-V P$}

Here we prove that, if an appropriate scalarization technique is chosen, the solutions of $R C-S_{\varphi}-V P$ coincide with the robust (strictly) efficient solutions of the original uncertain problem $V P(\mathcal{U})$. Hence, the proposed scalarization scheme provides necessary and sufficient robust optimality conditions. Let $F\left(x_{0}\right) \in \mathcal{F}$ be nonempty, $K$-proper and $K$-closed. Let us consider problem $S_{\varphi}-V P(\mathcal{U})$, obtained through scalarization by means of the function $\varphi_{F\left(x_{0}\right)-K}$.

\section{Robust strict efficiency}

A correspondence between a robust strictly efficient element, whose image set is the unique element in its equivalence class, and the unique solution of the robust counterpart of the scalarization is established here.

Sufficient robust strict optimality conditions by means of the robust counterpart of the scalarized uncertain vector problem are provided under appropriate assumptions on the function $\varphi$ involved in the objective of problem $S_{\varphi}-V P(\mathcal{U})$.

Proposition 4.8 Let $F\left(x_{0}\right) \in \mathcal{F}$ be nonempty, $K$-proper and $K$-closed and let $A=F\left(x_{0}\right)$ in the formulation of $R C-S_{\varphi}-V P$. Let $\varphi$ be $\leq$-preserving and strictly $\leq$-representing at 0 on $Y$ and let property $(P)$ hold. If the element $x_{0} \in \mathcal{X}$ is the unique solution of $R C-S_{\varphi}-V P$, then it is robust strictly efficient. 
Proof The thesis follows from Proposition 3.1, since $\Phi_{F\left(x_{0}\right)-K}$ is $\prec$-preserving at $F\left(x_{0}\right)$ on $\mathcal{F}$ by Lemma 4.6.

In the same vein, we can obtain necessary robust strict optimality conditions.

Proposition 4.9 Let $F\left(x_{0}\right) \in \mathcal{F}$ be nonempty and let $A=F\left(x_{0}\right)$ in the formulation of $R C-S_{\varphi}-V P$. Let $\varphi$ be $\leq$-preserving and strictly $\leq$-representing at 0 on $Y$ and let property $(P)$ hold. If the element $x_{0} \in \mathcal{X}$ is robust strictly efficient and Assumption 4.2 is fulfilled at $F\left(x_{0}\right)$, then $x_{0}$ is the unique solution of problem $R C-S_{\varphi}-V P$.

Proof The thesis follows from Proposition 3.2, since $\Phi_{F\left(x_{0}\right)-K}$ is strictly $\prec$-representing at $F\left(x_{0}\right)$ on $\mathcal{F}$ by Lemma 4.7 .

\section{Robust efficiency}

A correspondence between a robust efficient element and a notion of optimal solution of the robust counterpart of the scalarization is established here. Such an approach allows us to consider problems where the equivalence class of the image of a given robust efficient element may not be a singleton in $2^{Y}$. In this framework, the formulation of optimality conditions through scalarization necessarily relies on the structure of the quotient set.

Sufficient robust optimality conditions formulated in the following Proposition by means of the robust counterpart of the scalarized uncertain vector problem are provided under appropriate assumptions on the function $\varphi$ involved in the objective of problem $S_{\varphi}-V P(\mathcal{U})$.

Proposition 4.10 Let $F\left(x_{0}\right) \in \mathcal{F}$ be nonempty, $K$-proper and $K$-closed and let $A=F\left(x_{0}\right)$ in the formulation of $R C-S_{\varphi}-V P$. Let $\varphi$ be $\leq$-preserving and strictly $\leq$-representing at 0 on $Y$ and let property $(P)$ hold. If

$$
\Phi_{F\left(x_{0}\right)-K}(F(x))>\Phi_{F\left(x_{0}\right)-K}\left(F\left(x_{0}\right)\right), \forall x \in \mathcal{X} \text { s.t. } F(x) \nsim F\left(x_{0}\right)
$$

then $x_{0}$ is robust efficient.

Proof Let $x \in \mathcal{X}$ be such that $F(x) \nsim F\left(x_{0}\right)$. The relation $\Phi_{F\left(x_{0}\right)-K}(F(x))>$ $\Phi_{F\left(x_{0}\right)-K}\left(F\left(x_{0}\right)\right)$ implies $F(x) \nprec F\left(x_{0}\right)$, since $\Phi_{F\left(x_{0}\right)-K}$ is $\prec$-preserving at $F\left(x_{0}\right)$ (see Lemma 4.6). Hence, for all $x \in \mathcal{X}$, it holds $F(x) \nprec F\left(x_{0}\right)$ or $F(x) \sim F\left(x_{0}\right)$, namely $x_{0}$ is robust efficient.

In the same vein we can obtain necessary robust optimality conditions.

Proposition 4.11 Let $F\left(x_{0}\right) \in \mathcal{F}$ be nonempty and let $A=F\left(x_{0}\right)$ in the formulation of $R C-S_{\varphi}-V P$. Let $\varphi$ be $\leq$-preserving and strictly $\leq$-representing at 0 on $Y$ and let property $(P)$ hold. If the element $x_{0} \in \mathcal{X}$ is robust efficient and Assumption 4.2 is fulfilled at $F\left(x_{0}\right)$, then

$$
\Phi_{F\left(x_{0}\right)-K}(F(x))>\Phi_{F\left(x_{0}\right)-K}\left(F\left(x_{0}\right)\right), \forall x \in \mathcal{X} \text { s.t. } F(x) \nsim F\left(x_{0}\right)
$$

Proof By contradiction, suppose that there exists $\bar{x} \in \mathcal{X} \backslash\left\{x_{0}\right\}$ such that $F(\bar{x}) \nsim F\left(x_{0}\right)$ and $\Phi_{F\left(x_{0}\right)-K}(F(\bar{x})) \leq \Phi_{F\left(x_{0}\right)-K}\left(F\left(x_{0}\right)\right)$. Since $\Phi_{F\left(x_{0}\right)-K}$ is strictly $\prec$-representing at $F\left(x_{0}\right)$ on $\mathcal{F}$ (see Lemma 4.7), the previous relation implies $F(\bar{x}) \prec F\left(x_{0}\right)$, a contradiction since $F(\bar{x}) \nsim F\left(x_{0}\right)$ and $x_{0}$ is robust efficient. 


\section{Equivalence between $R C-S_{\varphi}-V P$ and $S_{\psi}-R C-V P$.}

In this section, we emphasize a fundamental feature of the axiomatic scalarization techniques that we illustrated in the former Sections. Under appropriate conditions, we ensure that problems $S_{\Psi}-R C-V P$ and $R C-S_{\varphi}-V P$, introduced respectively in Sects. 3 and 4 as two alternative ways to obtain robust optimality conditions in vector optimization through scalarization, can be considered equivalent. On one hand, we obtain problem $S_{\Psi}-R C-V P$ from the set-valued robust counterpart $R C-V P$ by scalarization through the function $\Psi$. On the other hand, problem $R C-S_{\varphi}-V P$ is obtained as the robust counterpart of the scalarization based on the function $\varphi$ of the original uncertain vector problem $V P(\mathcal{U})$. The crucial result that allows us to commute robustification and scalarization is the fact that both $S_{\Psi}-R C-V P$ and $R C-S_{\varphi}-V P$ can be used to formulate necessary and sufficient robust optimality conditions. We underline that the two distinct scalarization processes $\Psi$ and $\varphi$ used respectively in $S_{\Psi}-R C-V P$ and $R C-S_{\varphi}-V P$ are independently chosen within an appropriate axiomatic approach.

\section{Robust strict efficiency}

The following Proposition shows that the element $x_{0} \in \mathcal{X}$ is robust strict efficient if and only if it is the unique solution of both problems $S_{\Psi}-R C-V P$ and $R C-S_{\varphi}-V P$.

Proposition 5.1 Let $F\left(x_{0}\right) \in \mathcal{F}$ be nonempty $K$-proper and $K$-closed and let $A=F\left(x_{0}\right)$ in the formulation of $R C-S_{\varphi}-V P$. Let $\Psi$ be $\prec$-preserving and strictly $\prec$-representing at $F\left(x_{0}\right)$ on $\mathcal{F}$. Moreover, let $\varphi$ be $\leq$-preserving and strictly $\leq$-representing at 0 on $Y$, let property $(P)$ hold and let Assumption 4.2 be fulfilled for $\varphi$ on $F\left(x_{0}\right)$. Then, the following statements are equivalent:

(1) the element $x_{0} \in \mathcal{X}$ is robust strictly efficient;

(2) the element $x_{0} \in \mathcal{X}$ is the unique solution of $S_{\Psi}-R C-V P$;

(3) the element $x_{0} \in \mathcal{X}$ is the unique solution of $R C-S_{\varphi}-V P$.

\section{Robust efficiency}

The following Proposition shows the existing connections between the solutions of problems $S_{\Psi}-R C-V P$ and $R C-S_{\varphi}-V P$ and the notion of robust efficiency. When we consider the optimality conditions on $R C-S_{\varphi}-V P$, the equivalence class of $F\left(x_{0}\right)$ should be taken into account.

Proposition 5.2 Let $F\left(x_{0}\right) \in \mathcal{F}$ be nonempty $K$-proper and $K$-closed and let $A=F\left(x_{0}\right)$ in the formulation of $R C-S_{\varphi}-V P$. Let $\Psi$ be strictly $\prec$-preserving and $\prec$-representing at $F\left(x_{0}\right)$ on $\mathcal{F}$. Moreover, let $\varphi$ be $\leq$-preserving and strictly $\leq$-representing at 0 on $Y$, let property $(P)$ hold and let Assumption 4.2 hold for $\varphi$ on $F\left(x_{0}\right)$. Then, the following statements are equivalent

(1) the element $x_{0} \in \mathcal{X}$ is robust efficient;

(2) the element $x_{0} \in \mathcal{X}$ is a solution of $S_{\Psi}-R C-V P$;

(3) $\Phi_{F\left(x_{0}\right)-K}(F(x))>\Phi_{F\left(x_{0}\right)-K}\left(F\left(x_{0}\right)\right), \forall x \in \mathcal{X}$ s.t. $F(x) \nsim F\left(x_{0}\right)$.

Remark 5.3 If, in Proposition 5.2, the map $\Psi$ is assumed to be そ-preserving and strictly $\prec$-representing at $F\left(x_{0}\right)$ on $\mathcal{F}$, point (2) can be replaced by the following: 
(2') $\Psi(F(x))>\Psi\left(F\left(x_{0}\right)\right), \forall x \in \mathcal{X}$ s.t. $F(x) \nsim F\left(x_{0}\right)$.

We conclude this Section with an example showing that the results presented in Proposition 5.1 do not hold without appropriate scalarization schemes. If we consider the notion of robust strict efficiency in vector optimization introduced in Sect. 3, Example 5.4 (see also example 1 in [4]) shows that, on one hand, not all the robust strictly efficient solutions of an original uncertain vector problem $V P(\mathcal{U})$ can be found by solving problem $R C-S_{\varphi}-V P$, when a linear scalarizing function is chosen as $\varphi$. Hence, linear scalarizations, even if widely used in applications, cannot be used in general to formulate problem $S_{\varphi}-V P(\mathcal{U})$ and to obtain necessary robust optimality conditions through $R C-S_{\varphi}-V P$. On the other hand, in Example 5.4 we formulate problem $S_{\Psi}-R C-V P$ choosing as $\Psi$ a (nonlinear) function based on the oriented distance, which fits into the axiomatic approach presented in Sect. 3. With this choice, the whole set of robust strictly efficient solutions of $V P(\mathcal{U})$ can be characterized through necessary and sufficient optimality conditions by solving $S_{\Psi}-R C-V P$. This shows that the commutativity of robustness and scalarization does not hold without an appropriate choice of $\Psi$ and $\varphi$.

Example 5.4 Let $Y=\mathbb{R}^{2}, K=\mathbb{R}_{+}^{2}, \mathcal{X}=[0,1]$ and $\mathcal{U}=\left\{\xi_{1}, \xi_{2}, \xi_{3}\right\}$. We consider the following uncertain vector optimization problem

$$
\operatorname{minimize} f(x, \xi) \text { s.t. } x \in[0,1] \quad V P_{E x(5.4)}(\mathcal{U})
$$

where the objective $f:[0,1] \times \mathcal{U} \rightarrow \mathbb{R}^{2}$ is defined in the three scenarios as follows

$$
\begin{aligned}
& f\left(x, \xi_{1}\right)=x\left(\begin{array}{ll}
0 & 2
\end{array}\right)^{T}+(1-x)(14)^{T} \\
& f\left(x, \xi_{2}\right)=x(22)^{T}+(1-x)\left(\begin{array}{ll}
1 & 1
\end{array}\right)^{T} \\
& f\left(x, \xi_{3}\right)=x(20)^{T}+(1-x)(41)^{T}
\end{aligned}
$$

\section{Step 1. (robust strictly efficient solutions)}

Let us show that any element in $[0,1]$ is robust strictly efficient, according to Definition 2.9. Indeed, fix $x_{0} \in[0,1]$. If $x \in\left(x_{0}, 1\right]$, then the relation $f\left(x, \xi_{2}\right) \not \leq f\left(x_{0}, \xi_{i}\right)$ holds for all $i=1,2,3$. Similarly, if $x^{\prime \prime} \in[0, x)$, then the relation $f\left(x^{\prime \prime}, \xi_{1}\right) \not \leq f\left(x, \xi_{i}\right)$ holds for all $i=1,2,3$. Hence, any $x_{0} \in[0,1]$ is robust strictly efficient.

Step 2. (Formulation of problem $R C-S_{\varphi}-V P$ )

Problem $S_{\Lambda}-V P_{E x(5.4)}(\mathcal{U})$ is obtained from $V P_{E x(5.4)}(\mathcal{U})$ by linear scalarization with the linear function $\Lambda: \mathbb{R}^{2} \rightarrow \mathbb{R}$, where $\Lambda\left(x_{1}, x_{2}\right)=\lambda x_{1}+(1-\lambda) x_{2}$ and $\lambda \in[0,1]$. Its robust counterpart is

$$
\operatorname{minimize} \max _{\xi \in \mathcal{U}} \Lambda(f(x, \xi)) \text { s.t. } x \in[0,1] \quad R C-S_{\Lambda}-V P_{E x(5.4)}
$$

We show that the robust counterpart $R C-S_{\Lambda}-V P_{E x(5.4)}$ cannot be used to provide necessary robust optimality conditions. Indeed, the explicit computation of $\max _{\xi \in \mathcal{U}} \Lambda \circ f(0, \xi)$ and $\max _{\xi \in \mathcal{U}} \Lambda \circ f(1, \xi)$ yields

$$
\max _{\xi \in \mathcal{U}} \Lambda \circ f(0, \xi) \in[5 / 2,4] \text { and } \max _{\xi \in \mathcal{U}} \Lambda \circ f(1, \xi)=2
$$

Hence, $x=0$ cannot be a solution of $R C-S_{\Lambda}-V P$ for any linear function $\Lambda$, even if $x=0$ is a robust strictly efficient element ${ }^{1}$.

\footnotetext{
1 It is easy to verify that $\arg \min _{x \in[0,1]} \max _{\xi \in \mathcal{U}} \Lambda(f(x, \xi)) \geq 3 / 5$ for all $\lambda \in[0,1]$. Therefore, any $x \in$ $[0,3 / 5)$ cannot be a solution of $R C-S_{\Lambda}-V P$, for any linear function $\Lambda$, even if the points in $[0,3 / 5)$ are in the set of robust strictly efficient elements
} 


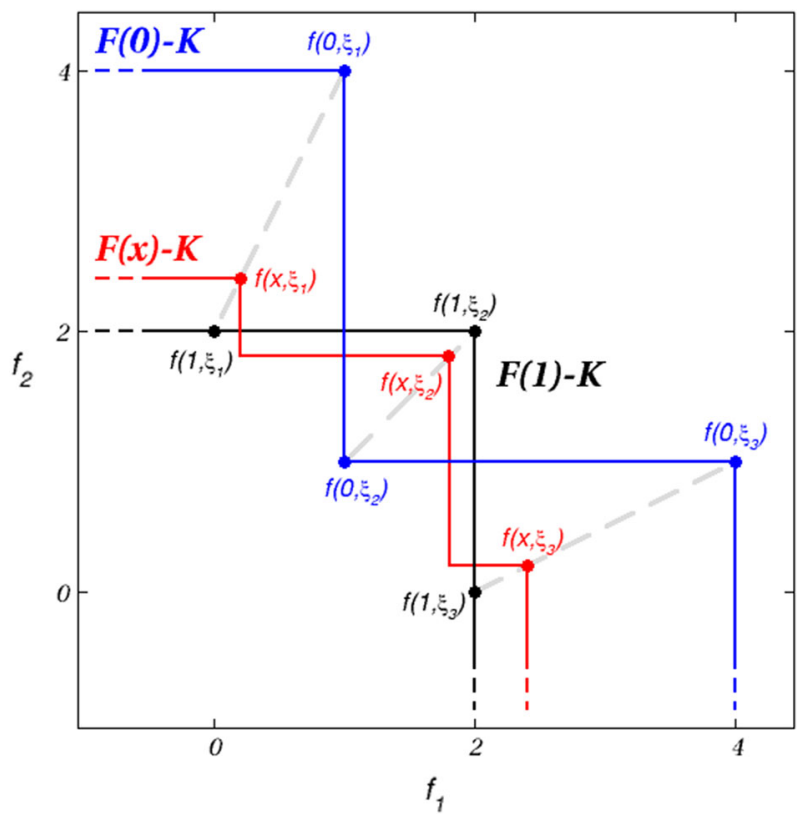

Fig. 3 Set-valued images of map $F$ at $x=0$ (blue), $x=1$ (black) and generic $x \in(0,1)$ (red)

Step 3. (Formulation of problem $S_{\Psi}-R C-V P$ )

The set-valued robust counterpart of problem $V P_{E x(5.4)}(\mathcal{U})$ is

$$
\prec-\operatorname{minimize} F(x) \text { s.t. } x \in[0,1] \quad R C-V P_{E x(5.4)}
$$

where the set-valued map $F:[0,1] \rightarrow 2^{\mathbb{R}^{2}}$ is

$$
F(x)=\bigcup_{i=1}^{3} f\left(x, \xi_{i}\right)=\left\{\left(\begin{array}{c}
1-x \\
4-2 x
\end{array}\right),\left(\begin{array}{c}
x+1 \\
x+1
\end{array}\right),\left(\begin{array}{c}
4-2 x \\
1-x
\end{array}\right)\right\}, x \in[0,1]
$$

In Fig. 3 a representation of some set-valued images of map $F$ is provided.

We choose as scalarizing function $\Psi$ the function $\Delta_{A}: \mathcal{F} \rightarrow \mathbb{R}$ defined as

$$
\Delta_{A}(B)=\sup _{b \in B} d(b, A-K)-\inf _{b \in B} d(b, Y \backslash(A-K)), \quad B \in \mathcal{F}
$$

where $A \in \mathcal{F}$ is nonempty and $d\left(y, B^{\prime}\right)=\inf \left\{\left\|y-b^{\prime}\right\|: b^{\prime} \in B^{\prime}\right\}$ is the distance of the element $y \in Y$ from a (nonempty) set $B^{\prime}$. We mention that this function is considered in [13] and it is an extension of the oriented distance function considered in [31] to a set-valued framework. With this choice of $\Psi$, the scalar problem $S_{\Delta}-R C-V P_{E x(5.4)}$ is

$$
\text { minimize } \Delta_{A}(F(x)) \text { s.t. } x \in[0,1] \quad S_{\Delta}-R C-V P_{E x(5.4)}
$$

Let us consider the element $x_{0} \in[0,1]$ and let $A=F\left(x_{0}\right)$. If $x_{0}$ is robust strictly efficient, then for each $x \in[0,1]$, with $x \neq x_{0}$, an element $y \in F(x)$ exists such that $y \in Y \backslash\left(F\left(x_{0}\right)-K\right)$. Hence, inf $y_{y^{\prime} \in F(x)} d\left(y^{\prime}, Y \backslash\left(F\left(x_{0}\right)-K\right)\right)=0$ and

$$
\Delta_{F\left(x_{0}\right)}(F(x))=\sup _{y^{\prime} \in F(x)} d\left(y^{\prime}, F\left(x_{0}\right)-K\right) \geq d\left(y, F\left(x_{0}\right)-K\right)>0
$$


Since $\Delta_{F\left(x_{0}\right)}\left(F\left(x_{0}\right)\right)=0$ (see Proposition 6.6 in [13]), it follows that

$$
\Delta_{F\left(x_{0}\right)}(F(x))>\Delta_{F\left(x_{0}\right)}\left(F\left(x_{0}\right)\right)
$$

holds, for all $x \in[0,1]$ with $x \neq x_{0}$. Hence, $x_{0}$ is the unique solution of problem $S_{\Delta}-R C-$ $V P_{E x(5.4)}$. Conversely, suppose that $x_{0} \in[0,1]$ is the unique solution of problem $S_{\Delta}-R C-$ $V P_{E x(5.4)}$. By contradiction, suppose that $x_{0}$ is not robust strictly efficient. Hence, an $x \in$ $[0,1]$ with $x \neq x_{0}$ exists such that $F(x) \prec F\left(x_{0}\right)$. Then, $\sup _{y^{\prime} \in F(x)} d\left(y^{\prime}, F\left(x_{0}\right)-K\right)=0$ holds, which implies $\Delta_{F\left(x_{0}\right)}(F(x))=-\inf _{y^{\prime} \in F(x)} d\left(y^{\prime}, Y \backslash\left(F\left(x_{0}\right)-K\right)\right) \leq 0$. It follows that $x_{0}$ is not the unique solution of problem $S_{\Delta}-R C-V P_{E x(5.4)}$ since $\Delta_{F\left(x_{0}\right)}\left(F\left(x_{0}\right)\right)=0$, a contradiction.

Step 4. (Comparison of $S_{\Psi}-R C-V P$ and $R C-S_{\varphi}-V P$ )

While with $S_{\Delta}-R C-V P_{E x(5.4)}$ we obtain the whole set $[0,1]$ of robust strictly efficient solutions of $V P_{E x(5.4)}(\mathcal{U})$, with $R C-S_{\Lambda}-V P_{E x(5.4)}$ the robust strictly efficient solutions $x_{0} \in[0,3 / 5$ ) cannot be found (see the footnote 1 in Step 2, where we omit details of the calculations). In particular, we explicitly show that the robust strictly efficient element $x_{0}=0$ is a solution of $S_{\Delta}-R C-V P_{E x(5.4)}$ and not a solution of $R C-S_{\Lambda}-V P_{E x(5.4)}$.

\section{Some special cases}

In this Section we consider two examples of scalarization methods that are based respectively on the Gerstewitz and the oriented distance functions. Similar approaches to scalarization are widely used in vector optimization (see, e.g., [20] or [29]). Both the approaches are placed within the theoretical framework outlined in Sect. 4, where the scalarizing functions are characterized by the structure defined in (3). Here, the scalarization procedure is applied to an uncertain vector problem $V P(\mathcal{U})$, hence an uncertain scalar problem $S_{\varphi}-V P(\mathcal{U})$ is obtained. Through the direct application of the robust approach introduced in [2], a deterministic scalar robust counterpart $R C-S_{\varphi}-V P$ can be formulated, whose solutions are coherent with the set-valued approach along the line marked by [6]. Such a process allows us to avoid the explicit study of the set-valued robust counterpart $R C-V P$ of the uncertain vector problem $V P(\mathcal{U})$ and its scalarization $S_{\Psi}-R C-V P$, whose formulation may remain implicit due to the commutativity property outlined in Sect. 5 .

\subsection{The Gerstewitz approach}

Let $Y$ be a topological vector space. Provided that the ordering cone $K \subseteq Y$ has nonempty interior, the so called Gerstewitz function $\phi_{e, a}: Y \rightarrow \mathbb{R}$ is defined by

$$
\phi_{e, a}(y)=\min \{t \in \mathbb{R}: y \in t e+a-K\}
$$

where $e \in \operatorname{int} K$ is a fixed element and $a \in Y$ (see e.g. [9]). We remark that in the special case of multiobjective optimization, in which $Y=\mathbb{R}^{p}$ and $K=\mathbb{R}_{+}^{p}$, several well known scalarization methods, such as Pascoletti-Serafini, $\epsilon$-constraint, Chebyshev scalarization, among others, can be reduced to the use of the Gerstewitz scalarizing function (see e.g. Sect. 2.5 in [7]).

\section{Formulation of problem $R C-S_{\varphi}-V P$}

We consider the robustification-scalarization approach outlined in the right hand side of Fig. 1. When we chose $\varphi$ as the Gerstewitz function $\phi_{e, a}$ within the axiomatic approach 
presented in Sect. 4, we obtain $\phi_{e, A-K}: Y \rightarrow \mathbb{R}$ as

$$
\phi_{e, A-K}(y)=\inf _{a \in A-K} \phi_{e, 0}(y-a), \quad \forall y \in Y
$$

where $A \subseteq Y$ is nonempty.

Remark 6.1 It is easy to verify that $\phi_{e, A-K}(y)=\phi_{e, A}(y)$ when $A$ is $K$-proper and $K$-closed. Indeed, by Proposition 3.2 in [14], it follows $\phi_{e, A}(y)=\min \{t \in \mathbb{R}: y \in t e+A-K\}$. Since $K$ is convex, it results

$$
\begin{aligned}
\phi_{e, A-K}(y) & =\min \{t \in \mathbb{R}: y \in t e+A-K-K\} \\
& =\min \{t \in \mathbb{R}: y \in t e+A-K\} \\
& =\phi_{e, A}(y)
\end{aligned}
$$

The function $\phi_{e, A}$ has many applications in the context of nonlinear analysis and it was used as a scalarizing function to obtain optimality conditions in vector optimization problems (see [20] and [29] and the references therein). Scalarizations through the Gerstewitz function have also been applied to consider robustness in set-valued frameworks (see e.g. [22] and [23]).

The scalarization of the uncertain vector problem $V P(\mathcal{U})$ through $\phi_{e, A-K}: Y \rightarrow \mathbb{R}$, where $A \in \mathcal{F}$, is

$$
\text { minimize } \phi_{e, A-K}(f(x, \xi)) \text { s.t. } x \in \mathcal{X} \quad S_{\phi}-V P(\mathcal{U})
$$

The robust counterpart of $S_{\phi}-V P(\mathcal{U})$ in the sense of [3] is

$$
\text { minimize } \sup _{\xi \in \mathcal{U}} \phi_{e, A-K}(f(x, \xi)) \text { s.t. } x \in \mathcal{X} \quad R C-S_{\phi}-V P
$$

We note that the function $\phi_{e, 0}$ is $\leq$-preserving and strictly $\leq$-representing at 0 on $Y$ and that property (P) is fulfilled for $\phi_{e, 0}$ (see [9], Theorem 2.1). Moreover, the following Lemma shows that, when the nonempty set $A$ is $K$-proper and $K$-closed, the infimum $\inf _{a \in A-K} \phi_{e, 0}(y-$ $a)=\phi_{e, A-K}(y)$ is attained on $\partial(A-K)$, for all $y \in Y$.

Lemma 6.2 Let $A \in \mathcal{F}$ be $K$-proper and $K$-closed. Then, for all $y \in Y$ there exists $\bar{a} \in$ $\partial(A-K)$ such that

$$
\phi_{e, A-K}(y)=\inf _{a^{\prime} \in A-K} \phi_{e, a^{\prime}}(y)=\phi_{e, 0}(y-\bar{a})
$$

Proof For any $t=\phi_{e, A-K}(y)$ it holds $y \in t e+\partial(A-K)$ (see [9] or [14]). Then, there exists $\bar{a} \in \partial(A-K)$ such that $y=t e+\bar{a}$. Hence,

$$
\phi_{e, 0}(y-\bar{a})=\phi_{e, 0}(t e)=t=\phi_{e, A-K}(y)=\inf _{a^{\prime} \in A-K} \phi_{e, a^{\prime}}(y)=\inf _{a^{\prime} \in A-K} \phi_{e, 0}\left(y-a^{\prime}\right)
$$

and the thesis follows.

From the above mentioned facts, Propositions 4.8 and 4.9 can be reformulated to provide necessary and suffcient robust strict optimality conditions.

Corollary 6.3 Let $F\left(x_{0}\right) \in \mathcal{F}$ be $K$-proper and $K$-closed and let $A=F\left(x_{0}\right)$ in the formulation of $R C-S_{\phi}-V P$. The element $x_{0} \in \mathcal{X}$ is robust strictly efficient if and only if $x_{0}$ is the unique solution of problem $R C-S_{\phi}-V P$.

Similarly, Propositions 4.10 and 4.11 can be reformulated to provide necessary and suffcient robust optimality conditions. 
Corollary 6.4 Let $F\left(x_{0}\right) \in \mathcal{F}$ be $K$-proper and $K$-closed and let $A=F\left(x_{0}\right)$ in the formulation of $R C-S_{\phi}-V P$. The element $x_{0} \in \mathcal{X}$ is robust efficient if and only if

$$
\sup _{\xi \in \mathcal{U}} \phi_{e, F\left(x_{0}\right)}(f(x, \xi))>\sup _{\xi \in \mathcal{U}} \phi_{e, F\left(x_{0}\right)}\left(f\left(x_{0}, \xi\right)\right), \forall x \in \mathcal{X} \text { s.t. } F(x) \nsim F\left(x_{0}\right)
$$

\section{Formulation of problem $S_{\Psi}-R C-V P$}

We consider the scalarization-robustification approach outlined in the left hand side of Fig. 1. Let $G_{e, A}: \mathcal{A} \rightarrow \mathbb{R}$ be the extension of the Gerstewitz function to a set-valued framework defined by

$$
G_{e, A}(B)=\inf \{t \in \mathbb{R}: B \subseteq t e+A-K\}, \quad B \in \mathcal{A}
$$

where $\mathcal{A}$ is a collection of nonempty $K$-proper and $K$-closed sets, $A \in \mathcal{A}$ and $e \in \operatorname{int} K$. This extention of the Gerstewitz function is considered in [13] and here it is adapted to be consistent with the upper type partial quasi order set relation “२”". Indeed, Proposition 4.11 in [13] can be adapted in this context, thus esuring that $G_{e, A}$ is $\prec$-preserving and strictly $\prec$-representing at $A$ on $\mathcal{A}$.

If we choose the scalarizing map $\Phi$ as $G_{e, A}$, the (set-valued) robust counterpart of the uncertain vector problem $V P(\mathcal{U})$ is

$$
\text { minimize } G_{e, A}(F(x)) \text { s.t. } x \in \mathcal{X} \quad S_{G}-R C-V P
$$

where $A \in \mathcal{F}$. Now, Propositions 3.1 and 3.2 can be reformulated to provide necessary and suffcient robust strict optimality conditions based on $G_{e, A}$.

Corollary 6.5 Let the elements of collection $\mathcal{F}$ be $K$-proper and $K$-closed sets and let $A=$ $F\left(x_{0}\right)$ in the formulation of $R C-S_{G}-V P$. The element $x_{0}$ is robust strictly efficient if and only if $x_{0}$ is the unique solution of $S_{G}-R C-V P$.

Moreover, we provide necessary and sufficient robust optimality conditions taking into account Remark 5.3.

Corollary 6.6 Let the elements of collection $\mathcal{F}$ be $K$-proper and $K$-closed sets and let $A=$ $F\left(x_{0}\right)$ in the formulation of $R C-S_{G}-V P$. The element $x_{0}$ is robust efficient if and only if

$$
G_{e, F\left(x_{0}\right)}(F(x))>G_{e, F\left(x_{0}\right)}\left(F\left(x_{0}\right)\right), \forall x \in \mathcal{X} \text { s.t. } F(x) \nsim F\left(x_{0}\right)
$$

To conclude, by Corollary 6.3 and 6.5 (resp. 6.4 and 6.6), the same set of robust strictly efficient (resp. robust efficient) solutions of an uncertain vector optimization problem $V P(\mathcal{U})$ can be characterized by necessary and sufficient optimality conditions. These conditions are (equivalently) obtained in this context through both the approaches described in Fig. 1, according to which problems $R C-S_{\phi}-V P$ and $S_{G}-R C-V P$ are formulated.

\subsection{The oriented distance approach}

An alternative approach, where $Y$ is a normed vector space and the ordering cone $K$ is not necessarily solid, is based on the oriented distance. The so called oriented distance function $\Delta_{S}: Y \rightarrow \mathbb{R}$ was introduced in [15], where $S \subseteq Y$ is nonempty. The oriented distance takes the following form:

$$
\Delta_{S}(y)=d_{S}(y)-d_{(Y \backslash S)}(y), \quad \forall y \in Y
$$


where $d_{S}(y)=\inf \{\|s-y\|: s \in S\}$ is the distance of the element $y \in Y$ from the nonempty set $S$ with respect to a given norm. The oriented distance has many applications in the context of nonlinear analysis and it was used as a scalarizing function to obtain optimality conditions in vector optimization problems. To this purpose, we refer the reader to [31], where important properties of the oriented distance are proved. See also [20] and the references therein.

\section{Formulation of problem $R C-S_{\varphi}-V P$}

We consider the robustification-scalarization approach outlined in the right hand side of Fig. 1. When we chose $\varphi$ as the oriented distance $\Delta_{-K}$ (obtained from $\Delta_{S}$ by choosing $S=-K$ ) within the axiomatic approach presented in Sect. 4, we obtain $\delta_{A-K}: Y \rightarrow \mathbb{R}$ as

$$
\delta_{A-K}(y)=\inf _{a \in A-K} \Delta_{-K}(y-a), \quad \forall y \in Y
$$

The scalarization of the uncertain vector problem $V P(\mathcal{U})$ through $\delta_{A-K}: Y \rightarrow \mathbb{R}$, where $A \in \mathcal{F}$, reads as

$$
\text { minimize } \delta_{A-K}(f(x, \xi)) \text { s.t. } x \in \mathcal{X} \quad S_{\delta}-V P(\mathcal{U})
$$

The robust counterpart of $S_{\delta}-V P(\mathcal{U})$ in the sense of [3] results

$$
\operatorname{minimize} \sup _{\xi \in \mathcal{U}} \delta_{A-K}(f(x, \xi)) \text { s.t. } x \in \mathcal{X} \quad R C-S_{\delta}-V P
$$

We note that the oriented distance $\Delta_{-K}$ is $\leq$-preserving at 0 on $Y$ and property $(\mathrm{P})$ holds (see [31], Proposition 3.2 point (7) and (3) respectively).

Lemma 6.7 The function $\Delta_{-K}$ is strictly $\leq$-representing at 0 on $Y$.

Proof Let $y \in Y$ and let the relation $\Delta_{-K}(y) \leq \Delta_{-K}(0)$ hold. Since $0 \in \partial(-K)$, then $\Delta_{-K}(0)=0$. Hence $\Delta_{-K}(y) \leq 0$ holds, which implies $y \in-K$ (see [31], Proposition 3.2 point (3)), namely $y \leq 0$.

In the specific case where $\varphi_{A-K}=\delta_{A-K}$, point 3) in Lemma 4.4 can be proved omitting Assumption 4.2. To show this, the following relation between $\delta_{A-K}$ and $\Delta_{A-K}$ will be used.

Lemma 6.8 Let $A \subseteq Y$ be nonempty and $K$-proper and let $y \notin \operatorname{int}(A-K)$. Then $\delta_{A-K}(y)=$ $\Delta_{A-K}(y)$.

Proof Since $y \notin \operatorname{int}(A-K)$, then $y-a \notin \operatorname{int}(-K)$ for all $a \in A-K$. Hence $d_{Y \backslash-K}(y-a)=$ 0 for all $a \in A-K$. If follows

$$
\begin{aligned}
\delta_{A-K}(y) & =\inf _{a \in A-K}\left(d_{-K}(y-a)-d_{Y \backslash-K}(y-a)\right) \\
& =\inf _{a \in A-K} d_{-K}(y-a) \\
& =\inf _{a \in A-K} \inf _{k \in-K}\|a+k-y\| \\
& =\inf _{a \in A-K} \inf _{z \in\{a\}-K}\|z-y\| \\
& =\inf _{z \in A-K}\|z-y\| \\
& =\Delta_{A-K}(y)
\end{aligned}
$$


The next Lemma shows that implication 3) in Lemma 4.4 holds for $\delta_{A-K}$ when $A$ is nonempty, $K$-proper and $K$-closed, with no need of Assumption 4.2.

Lemma 6.9 Let $A \subseteq Y$ be nonempty, $K$-proper and $K$-closed. Then

$$
\delta_{A-K}(y) \leq 0 \Longrightarrow y \in A-K
$$

Proof By contradiction, suppose that $\delta_{A-K}(y) \leq 0$ and $y \notin A-K$. Since $y \not \leq a$ for all $a \in A-K$, then $\Delta_{-K}(y-a)>0$ for all $a \in A-K$, since $\Delta_{-K}$ is strictly $\leq$-representing at 0 on $Y$. Hence, $\delta_{A-K}(y)=\inf _{a \in A-K} \Delta_{-K}(y-a) \geq 0$; therefore, $\delta_{A-K}(y)=0$ follows. This equality, together with Lemma 6.8, leads to $\delta_{A-K}(y)=\Delta_{A-K}(y)=0$, which implies $y \in \partial(A-K)$ (see [31], Proposition 3.2 point 3), a contradiction since $A$ is $K$-closed.

From the above mentioned facts, Propositions 4.8 and 4.9 can be reformulated to provide necessary and sufficient robust strict optimality conditions.

Corollary 6.10 Let $F\left(x_{0}\right) \in \mathcal{F}$ be $K$-proper and $K$-closed and let $A=F\left(x_{0}\right)$ in the formulation of $R C-S_{\delta}-V P$. The element $x_{0} \in \mathcal{X}$ is robust strictly efficient if and only if $x_{0}$ is the unique solution of problem $R C-S_{\delta}-V P$.

Similarly, Propositions 4.10 and 4.11 can be reformulated to provide necessary and sufficient robust optimality conditions.

Corollary 6.11 Let $F\left(x_{0}\right) \in \mathcal{F}$ be $K$-proper and $K$-closed and let $A=F\left(x_{0}\right)$ in the formulation of $R C-S_{\phi}-V P$. The element $x_{0} \in \mathcal{X}$ is robust efficient if and only if

$$
\sup _{\xi \in \mathcal{U}} \delta_{F\left(x_{0}\right)}(f(x, \xi))>\sup _{\xi \in \mathcal{U}} \delta_{F\left(x_{0}\right)}\left(f\left(x_{0}, \xi\right)\right), \forall x \in \mathcal{X} \text { s.t. } F(x) \nsim F\left(x_{0}\right)
$$

\section{Formulation of problem $S_{\Psi}-R C-V P$}

We consider the scalarization-robustification approach outlined in the left hand side of Fig. 1. We choose $\Psi$ as an extension of the oriented distance function to a set-valued framework, which is the map $\Delta_{A}: \mathcal{A} \rightarrow \mathbb{R}$ considered in Example 5.4 (see 9) defined by

$$
\Delta_{A}(B)=\sup _{b \in B} d(b, A-K)-\inf _{b \in B} d(b, Y \backslash(A-K)), \quad B \in \mathcal{A}
$$

where $\mathcal{A}$ is a collection of nonempty $K$-proper and $K$-closed sets and $A \in \mathcal{A}$. This extention of the oriented distance is considered in [13] (see also [18] or [19]) and here it is reframed to be consistent with the upper type partial quasi order set relation " $\prec$ ". Indeed, Proposition 6.6 in [13] can be adapted in this context, thus esuring that $\Delta_{A}$ is $\prec$-preserving and strictly $\prec$-representing at $A$ on $\mathcal{A}$.

The scalarization through function $\Delta_{A}$ of the (set-valued) robust counterpart in the sense of [6] of the uncertain vector problem $V P(\mathcal{U})$ is

$$
\text { minimize } \Delta_{A}(F(x)) \text { s.t. } x \in \mathcal{X} \quad S_{\Delta}-R C-V P
$$

where $A \in \mathcal{F}$. From the above mentioned facts, Propositions 3.1 and 3.2 can be reformulated to provide necessary and suffcient robust strict optimality conditions.

Corollary 6.12 Let the elements of collection $\mathcal{F}$ be $K$-proper and $K$-closed sets and let $A=F\left(x_{0}\right)$ in the formulation of $R C-S_{\Delta}-V P$. The element $x_{0}$ is robust strictly efficient if and only if $x_{0}$ is the unique solution of $S_{\Delta}-R C-V P$. 
Moreover, we provide necessary and sufficient robust optimality conditions taking into account Remark 5.3.

Corollary 6.13 Let the elements of collection $\mathcal{F}$ be $K$-proper and $K$-closed sets and let $A=F\left(x_{0}\right)$ in the formulation of $R C-S_{\Delta}-V P$. The element $x_{0}$ is robust efficient if and only if

$$
\Delta_{F\left(x_{0}\right)}(F(x))>\Delta_{F\left(x_{0}\right)}\left(F\left(x_{0}\right)\right), \forall x \in \mathcal{X} \text { s.t. } F(x) \nsim F\left(x_{0}\right)
$$

To conclude, by Corollary 6.10 and 6.12 (resp. 6.11 and 6.13), the same set of robust strictly efficient (resp. robust efficient) solutions of an uncertain vector optimization problem $V P(\mathcal{U})$ can be characterised by necessary and sufficient optimality conditions. These conditions are (equivalently) obtained in this context through both the approaches described in Fig. 1, according to which problems $R C-S_{\delta}-V P$ and $S_{\Delta}-R C-V P$ are formulated.

\section{Conclusions}

In this work, we consider the non componentwise concepts of robust efficiency related to uncertain vector optimization problems, as introduced in [6] for multiobjective programs and extended by [16] for general spaces. Such robustness concepts can be reinterpreted within a set optimization framework. Indeed, a deterministic set-valued optimization problem $R C-V P$ can be formulated and interpreted as the robust counterpart of the original uncertain vector program $V P(\mathcal{U})$, where suitable solution concepts are coherent with notions of robust efficiency. When the deterministic set-valued robust counterpart is tackled through scalarization, we obtain the formulation of problem $S_{\Psi}-R C-V P$, where we identify the properties of the scalarizing functions $\Psi$ that are necessary and sufficient to fully characterize the robust optimal solutions. We show that necessary and sufficient robust optimality conditions can equivalently be provided by considering the robust counterpart $R C-S_{\varphi}-V P$ (in the sense of [2]) of the uncertain scalar problem $S_{\varphi}-V P(\mathcal{U})$, obtained through scalarization of the original uncertain vector program $V P(\mathcal{U})$. Since, under appropriate assumptions, both the approaches $S_{\Psi}-R C-V P$ and $R C-S_{\varphi}-V P$ provide necessary and sufficient robust optimality conditions, we claim that robustification and scalarization of an uncertain vector optimization problem $V P(\mathcal{U})$ can be swapped, with an independent choice of suitable maps $\Psi$ and $\varphi$. It follows that the transition to the set-valued robust counterpart $R C-V P$ may remain implicit, even if we a void the unduly pessimistic componentwise robustness notion in vector optimization. In fact, the application of the robust approach to the scalarized uncertain vector problem is proved to be fully coherent with the approach proposed by [6] and [16]. The use of a suitable scalarization of the parametric vector optimization problem directly provides robust optimality conditions, avoiding the explicit reference to a set optimization framework. Further research should study extensions of other notions of robustness to vector optimization (such as, for example, the concepts considered in $[11,16,22,23]$ ) and their behaviour with respect to the proposed scalarization scheme (see Fig. 1).

Acknowledgements The third author is partially supported by Ministerio de Ciencia e Innovacion (Spain), Project PGC2018-096899-B-I00, and by GNAMPA-indam (Italy). The authors thank the two anonymous referees, whose remarks helped to improve the quality of the work.

Open Access This article is licensed under a Creative Commons Attribution 4.0 International License, which permits use, sharing, adaptation, distribution and reproduction in any medium or format, as long as you give appropriate credit to the original author(s) and the source, provide a link to the Creative Commons licence, and indicate if changes were made. The images or other third party material in this article are included in the 
article's Creative Commons licence, unless indicated otherwise in a credit line to the material. If material is not included in the article's Creative Commons licence and your intended use is not permitted by statutory regulation or exceeds the permitted use, you will need to obtain permission directly from the copyright holder. To view a copy of this licence, visit http://creativecommons.org/licenses/by/4.0/.

\section{References}

1. Ansari, Q.H., Köbis, E., Sharma, P.K.: Characterizations of multiobjective robustness via oriented distance function and image space analysis. J. Optim. Theory Appl. 181, 817-839 (2019)

2. Ben-Tal, A., El Ghaoui, L., Nemirovski, A.: Robust Optimization. Princeton University Press, Princeton (2009)

3. Ben-Tal, A., Nemirovski, A.: Robust optimization-methodology and applications. Math. Program. 92(3), 453-480 (2002)

4. Bokrantz, R., Fredriksson, A.: Necessary and sufficient conditions for pareto efficiency in robust multiobjective optimization. Eur. J. Oper. Res. 262(2), 682-692 (2017)

5. Chen, J., Ansari, Q.H., Yao, J.-C.: Characterizations of set order relations and constrained set optimization problems via oriented distance function. Optimization 66(11), 1741-1754 (2017)

6. Ehrgott, M., Ide, J., Schöbel, A.: Minmax robustness for multi-objective optimization problems. Eur. J. Oper. Res. 239(1), 17-31 (2014)

7. Eichfelder, G.: Adaptive Scalarization Methods in Multiobjective Optimization. Springer, Berlin (2008)

8. Fliege, J., Werner, R.: Robust multiobjective optimization \& applications in portfolio optimization. Eur. J. Oper. Res. 234(2), 422-433 (2014)

9. Gerth, C., Weidner, P.: Nonconvex separation theorems and some applications in vector optimization. J. Optim. Theory Appl. 67(2), 297-320 (1990)

10. Goberna, M.A., Jeyakumar, V., Li, G., Vicente-Pérez, J.: Robust solutions to multi-objective linear programs with uncertain data. Eur. J. Oper. Res. 242(3), 730-743 (2015)

11. Groetzner, P., Werner, R.: Multiobjective optimization under uncertainty: a multiobjective robust (relative) regret approach. Eur. J. Oper. Res. 296, 101-115 (2021)

12. Gutiérrez, C., Huerga, L., Köbis, E., Tammer, C.: A scalarization scheme for binary relations with applications to set-valued and robust optimization. J. Global Optim. 79, 233-256 (2020)

13. Gutiérrez, C., Jiménez, B., Miglierina, E., Molho, E.: Scalarization in set optimization with solid and nonsolid ordering cones. J. Global Optim. 61(3), 525-552 (2015)

14. Hernández, E., Rodríguez-Marín, L.: Nonconvex scalarization in set optimization with set-valued maps. J. Math. Anal. Appl. 325(1), 1-18 (2007)

15. Hiriart-Urruty, J.-B.: Tangent cones, generalized gradients and mathematical programming in banach spaces. Math. Oper. Res. 4(1), 79-97 (1979)

16. Ide, J., Köbis, E., Kuroiwa, D., Schöbel, A., Tammer, C.: The relationship between multi-objective robustness concepts and set-valued optimization. Fixed Point Theory Appl. 2014(1), 83 (2014)

17. Ide, J., Schöbel, A.: Robustness for uncertain multi-objective optimization: a survey and analysis of different concepts. OR Spectrum 38(1), 235 (2016)

18. Jiménez, B., Novo, V., Vílchez, A.: A set scalarization function based on the oriented distance and relations with other set scalarizations. Optimization 67(12), 2091-2116 (2018)

19. Jiménez, B., Novo, V., Vílchez, A.: Characterization of set relations through extensions of the oriented distance. Math. Methods Oper. Res. 91(1), 89-115 (2020)

20. Khan, A., Tammer, C., Zalinescu, C.: Set-Valued Optimization. Springer, Berlin (2015)

21. Khushboo, Lalitha, C.S.: Scalarizations for a set optimization problem using generalized oriented distance function. Positivity 23, 1195-1213 (2019)

22. Klamroth, K., Köbis, E., Schöbel, A., Tammer, C.: A unified approach for different concepts of robustness and stochastic programming via non-linear scalarizing functionals. Optimization 62(5), 649-671 (2013)

23. Klamroth, K., Köbis, E., Schöbel, A., Tammer, C.: A unified approach to uncertain optimization. Eur. J. Oper. Res. 260(2), 403-420 (2017)

24. Köbis, E., Köbis, M.: Treatment of set order relations by means of a nonlinear scalarization functional: a full characterization. Optimization 65(10), 1805-1827 (2016)

25. Kuroiwa, D.: On set-valued optimization. Nonlinear Anal. Theory Methods Appl. 47(2), 1395-1400 (2001)

26. Kuroiwa, D.: Existence theorems of set optimization with set-valued maps. J. Inf. Optim. Sci. 24(1), 73-84 (2003) 
27. Kuroiwa, D., Lee, G.M.: On robust multiobjective optimization. Vietnam J. Math 40(2\&3), 305-317 (2012)

28. Soyster, A.L.: Convex programming with set-inclusive constraints and applications to inexact linear programming. Oper. Res. 21(5), 1154-1157 (1973)

29. Tammer, C., Weidner, P.: Scalarization and Separation by Translation Invariant Functions. Springer, Berlin (2020)

30. Wiecek, M., Dranichak, G.: Robust multiobjective optimization for decision making under uncertainty and conflict. In: Optimization Challenges in Complex, Networked and Risky Systems, pp. 84-114. INFORMS, (2016)

31. Zaffaroni, A.: Degrees of efficiency and degrees of minimality. SIAM J. Control Optim. 42(3), 1071-1086 (2003)

Publisher's Note Springer Nature remains neutral with regard to jurisdictional claims in published maps and institutional affiliations. 